\title{
On the decay and blow up of solutions for a quasilinear hyperbolic equations with nonlinear damping and source terms
}

\section{Erhan Pişkin*}

\section{"Correspondence:} episkin@dicle.edu.tr Department of Mathematics, Dicle University, Diyarbakir, Turkey

\begin{abstract}
In this work we investigate the global existence, decay, and blow up of solutions for a quasilinear hyperbolic equation. We prove the decay estimates of the energy function by using Nakao's inequality. Also, we obtain the blow up of solutions and lifespan estimates in three different ranges of the initial energy.
\end{abstract}

MSC: 35B40; 35B44; 35L72

Keywords: decay; blow up; quasilinear hyperbolic equation; nonlinear damping and source terms

\section{Introduction}

In this work we study the following quasilinear hyperbolic equations:

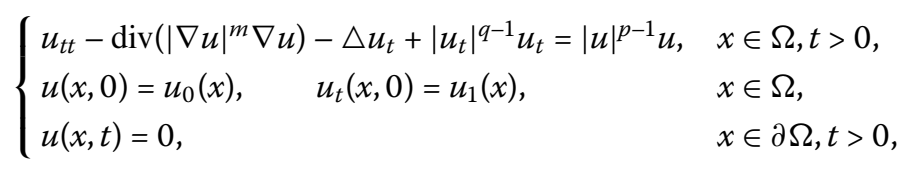

where $\Omega$ is a bounded domain with smooth boundary $\partial \Omega$ in $R^{n}(n \geq 1) ; m>0, p, q \geq 1$.

Problems of this type arise in physics. For example, this problem represents the longitudinal motion of a viscoelastic configuration which obeys a nonlinear Voight model [1,2].

When $m=0$, (1) becomes the following wave equation with nonlinear and strong damping terms:

$$
u_{t t}-\Delta u-\Delta u_{t}+\left|u_{t}\right|^{q-1} u_{t}=|u|^{p-1} u .
$$

Gerbi and Houari [3] studied the exponential decay, Chen and Liu [4] studied the global existence, decay, and exponential growth of solutions of the problem (2). Also, Gazzola and Squassina [5] studied the global existence and blow up of solutions of the problem (2), for $q=1$.

In the absence of the strong damping term $\Delta u_{t}$ and $m=0$, the problem (1) can be reduced to the following wave equation with nonlinear damping and source terms:

$$
u_{t t}-\Delta u+\left|u_{t}\right|^{q-1} u_{t}=|u|^{p-1} u
$$

(c) 2015 Pișkin. This article is distributed under the terms of the Creative Commons Attribution 4.0 International License (http://creativecommons.org/licenses/by/4.0/), which permits unrestricted use, distribution, and reproduction in any medium, provided you give appropriate credit to the original author(s) and the source, provide a link to the Creative Commons license, and indicate if changes were made. 
Many authors have investigated the local existence, blow up, and asymptotic behavior of solutions of (3); see [6-11]. The interaction between the damping $\left(\left|u_{t}\right|^{q-1} u_{t}\right)$ and the source term $\left(|u|^{p-1} u\right)$ makes the problem more interesting. Levine [7, 8] first studied the interaction between the linear damping $(q=1)$ and source term by using a concavity method. But this method cannot be applied in the case of a nonlinear damping term. Georgiev and Todorova [6] extended Levine's result to the nonlinear case $(q>1)$. They showed that solutions with a negative initial energy blow up in finite time. Later, Vitillaro [11] extended these results to the case of a nonlinear damping and a positive initial energy.

In [12], Messaoudi studied decay of solutions of the problem (1), using the techniques combination of the perturbed energy and potential well methods. Recently, the problem (1) was studied by Wu and Xue [13]. They proved the uniform energy decay rates of the solutions, by utilizing the multiplier method.

In this work, we established the polynomial and exponential decay of solutions of the problem (1) by using Nakao's inequality. After that, we show the blow up of solutions with negative and nonnegative initial energy, using the same techniques as in [14].

This work is organized as follows: In the next section, we present some lemmas, notations, and a local existence theorem. In Section 3, the global existence and decay of solutions are given. In Section 4, we show the blow up of solutions, for $q=1$.

\section{Preliminaries}

In this section, we shall give some assumptions and lemmas which will be used throughout this paper. Let $\|\cdot\|$ and $\|\cdot\|_{p}$ denote the usual $L^{2}(\Omega)$ norm and $L^{p}(\Omega)$ norm, respectively.

Lemma 1 (Sobolev-Poincaré inequality) [15] Let $p$ be a number with $2 \leq p<\infty(n=1,2)$ or $2 \leq p \leq \frac{2 n}{n-2}(n \geq 3)$, then there is a constant $C_{*}=C_{*}(\Omega, p)$ such that

$$
\|u\|_{p} \leq C_{*}\|\nabla u\| \quad \text { for } u \in H_{0}^{1}(\Omega) .
$$

Lemma 2 [16] Let $\phi(t)$ be a nonincreasing and nonnegative function defined on $[0, T]$, $T>1$, satisfying

$$
\phi^{1+\alpha}(t) \leq w_{0}(\phi(t)-\phi(t+1)), \quad t \in[0, T]
$$

for $w_{0}$ a positive constant and $\alpha$ a nonnegative constant. Then we have, for each $t \in[0, T]$,

$$
\begin{cases}\phi(t) \leq \phi(0) e^{-w_{1}[t-1]^{+}}, & \alpha=0, \\ \phi(t) \leq\left(\phi(0)^{-\alpha}+w_{0}^{-1} \alpha[t-1]^{+}\right)^{-\frac{1}{\alpha}}, & \alpha>0,\end{cases}
$$

where $[t-1]^{+}=\max \{t-1,0\}$ and $w_{1}=\ln \left(\frac{w_{0}}{w_{0}-1}\right)$.

Next, we state the local existence theorem that can be established by combining the arguments of $[6,17,18]$.

Theorem 3 (Local existence) Suppose that $m+2<p+1<\frac{n(m+2)}{n-(m+2)}, m+2<n$, and further $u_{0} \in W_{0}^{1, m+2}(\Omega)$ and $u_{1} \in L^{2}(\Omega)$ such that problem (1) has a unique local solution,

$$
u \in C\left([0, T) ; W_{0}^{1, m+2}(\Omega)\right) \quad \text { and } \quad u_{t} \in C\left([0, T) ; L^{2}(\Omega)\right) \cap L^{q+1}(\Omega \times[0, T)) .
$$


Moreover, at least one of the following statements holds true:

(i) $T=\infty$,

(ii) $\left\|u_{t}\right\|^{2}+\|\nabla u\|_{m+2}^{m+2} \rightarrow \infty$ as $t \rightarrow T^{-}$.

\section{Global existence and decay of solutions}

In this section, we discuss the global existence and decay of the solution for problem (1). We define

$$
J(t)=\frac{1}{m+2}\|\nabla u\|_{m+2}^{m+2}-\frac{1}{p+1}\|u\|_{p+1}^{p+1}
$$

and

$$
I(t)=\|\nabla u\|_{m+2}^{m+2}-\|u\|_{p+1}^{p+1} .
$$

We also define the energy function as follows:

$$
E(t)=\frac{1}{2}\left\|u_{t}\right\|^{2}+\frac{1}{m+2}\|\nabla u\|_{m+2}^{m+2}-\frac{1}{p+1}\|u\|_{p+1}^{p+1} .
$$

Finally, we define

$$
W=\left\{u: u \in W_{0}^{1, m+2}(\Omega), I(u)>0\right\} \cup\{0\} .
$$

The next lemma shows that our energy functional (6) is a nonincreasing function along the solution of (1).

Lemma $4 E(t)$ is a nonincreasing function for $t \geq 0$ and

$$
E^{\prime}(t)=-\left(\left\|u_{t}\right\|_{q+1}^{q+1}+\left\|\nabla u_{t}\right\|^{2}\right) \leq 0 .
$$

Proof Multiplying the equation of (1) by $u_{t}$ and integrating over $\Omega$, using integrating by parts, we get

$$
E(t)-E(0)=-\int_{0}^{t}\left(\left\|u_{\tau}\right\|_{q+1}^{q+1}+\left\|\nabla u_{\tau}\right\|^{2}\right) d \tau \quad \text { for } t \geq 0 .
$$

Lemma 5 Let $u_{0} \in W$ and $u_{1} \in L^{2}(\Omega)$. Suppose that $p>m+1$ and

$$
\beta=C_{*}\left(\frac{(p+1)(m+2)}{p-m-1} E(0)\right)^{\frac{p-m-1}{m+2}}<1,
$$

then $u \in W$ for each $t \geq 0$.

Proof Since $I(0)>0$, it follows by the continuity of $u(t)$ that

$$
I(t)>0
$$

for some interval near $t=0$. Let $T_{m}>0$ be a maximal time, when (5) holds on $\left[0, T_{m}\right]$. 
From (4) and (5), we have

$$
\begin{aligned}
J(t) & =\frac{1}{p+1} I(t)+\frac{p-m-1}{(p+1)(m+2)}\|\nabla u\|_{m+2}^{m+2} \\
& \geq \frac{p-m-1}{(p+1)(m+2)}\|\nabla u\|_{m+2}^{m+2} .
\end{aligned}
$$

Thus, from (6) and $E(t)$ being nonincreasing by (8), we have

$$
\begin{aligned}
\|\nabla u\|_{m+2}^{m+2} & \leq \frac{(p+1)(m+2)}{p-m-1} J(t) \\
& \leq \frac{(p+1)(m+2)}{p-m-1} E(t) \\
& \leq \frac{(p+1)(m+2)}{p-m-1} E(0) .
\end{aligned}
$$

And so, exploiting Lemma 1, (10), and (12), we obtain

$$
\begin{aligned}
\|u\|_{p+1}^{p+1} & \leq C_{*}\|\nabla u\|^{p+1} \\
& \leq C_{*}\|\nabla u\|_{m+2}^{p+1} \\
& =C_{*}\|\nabla u\|_{m+2}^{p-m-1}\|\nabla u\|_{m+2}^{m+2} \\
& \leq C_{*}\left(\frac{(p+1)(m+2)}{p-m-1} E(0)\right)^{\frac{p-m-1}{m+2}}\|\nabla u\|_{m+2}^{m+2} \\
& =\beta\|\nabla u\|_{m+2}^{m+2} \\
& <\|\nabla u\|_{m+2}^{m+2} \quad \text { on } t \in\left[0, T_{m}\right] .
\end{aligned}
$$

Therefore, by using (5), we conclude that $I(t)>0$ for all $t \in\left[0, T_{m}\right]$. By repeating the procedure, $T_{m}$ is extended to $T$. The proof of Lemma 5 is completed.

Lemma 6 Let the assumptions of Lemma 5 hold. Then there exists $\eta_{1}=1-\beta$ such that

$$
\|u\|_{p+1}^{p+1} \leq\left(1-\eta_{1}\right)\|\nabla u\|_{m+2}^{m+2}
$$

Proof From (13), we get

$$
\|u\|_{p+1}^{p+1} \leq \beta\|\nabla u\|_{m+2}^{m+2}
$$

Let $\eta_{1}=1-\beta$, then we have the following result.

Remark 7 From Lemma 6, we can deduce that

$$
\|\nabla u\|_{m+2}^{m+2} \leq \frac{1}{\eta_{1}} I(t)
$$

Theorem 8 Suppose that $m+2<p+1<\frac{n(m+2)}{n-(m+2)}, m+2<n$ holds. Let $u_{0} \in W$ satisfying (10). Then the solution of problem (1) is global. 
Proof It is sufficient to show that $\left\|u_{t}\right\|^{2}+\|\nabla u\|_{m+2}^{m+2}$ is bounded independently of $t$. To achieve this we use (5) and (6) to obtain

$$
\begin{aligned}
E(0) & \geq E(t)=\frac{1}{2}\left\|u_{t}\right\|^{2}+\frac{1}{m+2}\|\nabla u\|_{m+2}^{m+2}-\frac{1}{p+1}\|u\|_{p+1}^{p+1} \\
& =\frac{1}{2}\left\|u_{t}\right\|^{2}+\frac{p-m-1}{(p+1)(m+2)}\|\nabla u\|_{m+2}^{m+2}+\frac{1}{p+1} I(t) \\
& \geq \frac{1}{2}\left\|u_{t}\right\|^{2}+\frac{p-m-1}{(p+1)(m+2)}\|\nabla u\|_{m+2}^{m+2}
\end{aligned}
$$

since $I(t) \geq 0$. Therefore,

$$
\left\|u_{t}\right\|^{2}+\|\nabla u\|_{m+2}^{m+2} \leq C E(0)
$$

where $C=\max \left\{2, \frac{(p+1)(m+2)}{p-m-1}\right\}$. Then by Theorem 3 , we have the global existence result.

Theorem 9 Suppose that $m+2<p+1<\frac{n(m+2)}{n-(m+2)}, m+2<n$, and (10) hold, and further $u_{0} \in W$. Thus, we have the following decay estimates:

$$
E(t) \leq \begin{cases}E(0) e^{-w_{1}[t-1]^{+}}, & \text {if } q=1, m=0 \\ \left(E(0)^{-\alpha}+C_{7}^{-1} \alpha[t-1]^{+}\right)^{-\frac{1}{\alpha}}, & \text { if } q>\frac{1}{m+1}\end{cases}
$$

where $w_{1}, \alpha$, and $C_{7}$ are positive constants which will be defined later.

Proof By integrating (8) over $[t, t+1], t>0$, we have

$$
E(t)-E(t+1)=D^{q+1}(t)
$$

where

$$
D^{q+1}(t)=\int_{t}^{t+1}\left(\left\|u_{\tau}\right\|_{q+1}^{q+1}+\left\|\nabla u_{\tau}\right\|^{2}\right) d \tau
$$

By virtue of (16) and Hölder's inequality, we observe that

$$
\int_{t}^{t+1} \int_{\Omega}\left|u_{t}\right|^{2} d x d t \leq|\Omega|^{\frac{q+1}{q+2}} D^{2}(t)=C D^{2}(t) .
$$

Hence, from (17), there exist $t_{1} \in\left[t, t+\frac{1}{4}\right]$ and $t_{2} \in\left[t+\frac{3}{4}, t+1\right]$ such that

$$
\left\|u_{t}\left(t_{i}\right)\right\| \leq C D(t), \quad i=1,2 .
$$

Multiplying (1) by $u$ and integrating it over $\Omega \times\left[t_{1}, t_{2}\right]$, using integration by parts, we get

$$
\begin{aligned}
\int_{t_{1}}^{t_{2}} I(t) d t= & -\int_{t_{1}}^{t_{2}} \int_{\Omega} u u_{t t} d x d t-\int_{t_{1}}^{t_{2}} \int_{\Omega} \nabla u_{t} \nabla d x d t \\
& -\int_{t_{1}}^{t_{2}} \int_{\Omega}\left|u_{t}\right|^{q-1} u_{t} u d x d t .
\end{aligned}
$$


By using (1) and integrating by parts and applying the Cauchy-Schwarz inequality in the first term and the Hölder inequality in the second term of the right-hand side of (19), we obtain

$$
\begin{aligned}
\int_{t_{1}}^{t_{2}} I(t) d t \leq & \left\|u_{t}\left(t_{1}\right)\right\|\left\|u\left(t_{1}\right)\right\|+\left\|u_{t}\left(t_{2}\right)\right\|\left\|u\left(t_{2}\right)\right\| \\
& +\int_{t_{1}}^{t_{2}}\left\|u_{t}(t)\right\|^{2} d t+\int_{t_{1}}^{t_{2}}\left\|\nabla u_{t}\right\|\|\nabla u\| d t \\
& -\int_{t_{1}}^{t_{2}} \int_{\Omega}\left|u_{t}\right|^{q-1} u_{t} u d x d t .
\end{aligned}
$$

Now, our goal is to estimate the last term in the right-hand side of inequality (20). By using Hölder inequality, we obtain

$$
\int_{t_{1}}^{t_{2}} \int_{\Omega}\left|u_{t}\right|^{q-1} u_{t} u d x d t \leq \int_{t_{1}}^{t_{2}}\left\|u_{t}(t)\right\|_{q+1}^{q}\|u(t)\|_{q+1} d t
$$

By applying the Sobolev-Poincaré inequality and (12), we find

$$
\begin{aligned}
& \int_{t_{1}}^{t_{2}}\left\|u_{t}(t)\right\|_{q+1}^{q}\|u(t)\|_{q+1} d t \\
& \quad \leq C_{*} \int_{t_{1}}^{t_{2}}\left\|u_{t}(t)\right\|_{q+1}^{q}\|\nabla u\| d t \\
& \quad \leq C_{*} \int_{t_{1}}^{t_{2}}\left\|u_{t}(t)\right\|_{q+1}^{q}\|\nabla u\|_{m+2} d t \\
& \quad \leq C_{*}\left(\frac{(p+1)(m+2)}{p-m-1} E(0)\right)^{\frac{1}{m+2}} \int_{t_{1}}^{t_{2}}\left\|u_{t}(t)\right\|_{q+1}^{q} E^{\frac{1}{m+2}}(s) d t \\
& \quad \leq C_{*}\left(\frac{(p+1)(m+2)}{p-m-1} E(0)\right)^{\frac{1}{m+2}} \sup _{t_{1} \leq s \leq t_{2}} E^{\frac{1}{m+2}}(s) \int_{t_{1}}^{t_{2}}\left\|u_{t}(t)\right\|_{q+1}^{q} d t \\
& \quad \leq C_{*}\left(\frac{(p+1)(m+2)}{p-m-1} E(0)\right)^{\frac{1}{m+2}} \sup _{t_{1} \leq s \leq t_{2}} E^{\frac{1}{m+2}}(s) D^{q}(t) .
\end{aligned}
$$

Now, we estimate the fourth term of the right-hand side of inequality (20). By using the embedding $L^{m+2}(\Omega) \hookrightarrow L^{2}(\Omega)$, we have

$$
\begin{aligned}
& \int_{t_{1}}^{t_{2}}\left\|\nabla u_{t}\right\|\|\nabla u\| d t \\
& \quad \leq C_{*} \int_{t_{1}}^{t_{2}}\left\|\nabla u_{t}\right\|\|\nabla u(t)\|_{m+2} d t \\
& \quad \leq C_{*}\left(\frac{(p+1)(m+2)}{p-m-1} E(0)\right)^{\frac{1}{m+2}} \int_{t_{1}}^{t_{2}}\left\|\nabla u_{t}\right\| E^{\frac{1}{m+2}}(s) d t \\
& \quad \leq C_{*}\left(\frac{(p+1)(m+2)}{p-m-1} E(0)\right)^{\frac{1}{m+2}} \sup _{t_{1} \leq s \leq t_{2}} E^{\frac{1}{m+2}}(s) \int_{t_{1}}^{t_{2}}\left\|\nabla u_{t}\right\| d t,
\end{aligned}
$$


which implies

$$
\begin{aligned}
\int_{t_{1}}^{t_{2}}\left\|\nabla u_{t}\right\| d t & \leq\left(\int_{t_{1}}^{t_{2}} 1 d t\right)^{\frac{1}{2}}\left(\int_{t_{1}}^{t_{2}}\left\|\nabla u_{t}\right\|^{2} d t\right)^{\frac{1}{2}} \\
& \leq C D(t) .
\end{aligned}
$$

Then

$$
\int_{t_{1}}^{t_{2}}\left\|\nabla u_{t}\right\|\|\nabla u\| d t \leq C C_{*}\left(\frac{(p+1)(m+2)}{p-m-1} E(0)\right)^{\frac{1}{m+2}} \sup _{t_{1} \leq s \leq t_{2}} E^{\frac{1}{m+2}}(s) D(t) .
$$

From (12), (18), and the Sobolev-Poincaré inequality, we have

$$
\left\|u_{t}\left(t_{i}\right)\right\|\left\|u\left(t_{i}\right)\right\| \leq C_{1} D(t) \sup _{t_{1} \leq s \leq t_{2}} E^{\frac{1}{m+2}}(s),
$$

where $C_{1}=2 C_{*}\left(\frac{(p+1)(m+2)}{p-m-1} E(0)\right)^{\frac{1}{m+2}}$. Then by (20)-(24) we have

$$
\begin{aligned}
\int_{t_{1}}^{t_{2}} I(t) d t \leq & C_{1} D(t) \sup _{t_{1} \leq s \leq t_{2}} E^{\frac{1}{m+2}}(s)+D^{2}(t) \\
& +C C_{*}\left(\frac{(p+1)(m+2)}{p-m-1} E(0)\right)^{\frac{1}{m+2}} \sup _{t_{1} \leq s \leq t_{2}} E^{\frac{1}{m+2}}(s) D(t) \\
& +C_{*}\left(\frac{(p+1)(m+2)}{p-m-1} E(0)\right)^{\frac{1}{m+2}} \sup _{t_{1} \leq s \leq t_{2}} E^{\frac{1}{m+2}}(s) D^{q}(t) .
\end{aligned}
$$

On the other hand, from (5), (6), and Remark 7, we obtain

$$
E(t) \leq \frac{1}{2}\left\|u_{t}\right\|^{2}+C_{3} I(t)
$$

where $C_{3}=\frac{1}{\eta} \frac{p-m-1}{(p+1)(m+2)}+\frac{1}{p+1}$.

By integrating (26) over $\left[t_{1}, t_{2}\right]$, we have

$$
\int_{t_{1}}^{t_{2}} E(t) d t \leq \frac{1}{2} \int_{t_{1}}^{t_{2}}\left\|u_{t}\right\|^{2} d t+C_{3} \int_{t_{1}}^{t_{2}} I(t) d t .
$$

Then by (18), (25), and (27), we get

$$
\begin{aligned}
\int_{t_{1}}^{t_{2}} E(t) d t \leq & \frac{1}{2} C D^{2}(t)+C_{3}\left[C_{1} D(t) \sup _{t_{1} \leq s \leq t_{2}} E^{\frac{1}{m+2}}(s)+D^{2}(t)\right. \\
& +C C_{*}\left(\frac{(p+1)(m+2)}{p-m-1} E(0)\right)^{\frac{1}{m+2}} \sup _{t_{1} \leq s \leq t_{2}} E^{\frac{1}{m+2}}(s) D(t) \\
& \left.+C_{*}\left(\frac{(p+1)(m+2)}{p-m-1} E(0)\right)^{\frac{1}{m+2}} \sup _{t_{1} \leq s \leq t_{2}} E^{\frac{1}{m+2}}(s) D^{q}(t)\right] .
\end{aligned}
$$

By integrating $(8)$ over $\left[t, t_{2}\right]$, we obtain

$$
E(t)=E\left(t_{2}\right)+\int_{t}^{t_{2}}\left(\left\|u_{\tau}\right\|_{q+1}^{q+1}+\left\|\nabla u_{\tau}\right\|^{2}\right) d \tau
$$


Therefore, since $t_{2}-t_{1} \geq \frac{1}{2}$, we conclude that

$$
\int_{t_{1}}^{t_{2}} E(t) d t \geq\left(t_{2}-t_{1}\right) E\left(t_{2}\right) \geq \frac{1}{2} E\left(t_{2}\right)
$$

That is,

$$
E\left(t_{2}\right) \leq 2 \int_{t_{1}}^{t_{2}} E(t) d t
$$

Consequently, exploiting (15), (28)-(30), and since $t_{1}, t_{2} \in[t, t+1]$, we get

$$
\begin{aligned}
E(t) & \leq 2 \int_{t_{1}}^{t_{2}} E(t) d t+\int_{t}^{t+1}\left(\left\|u_{\tau}\right\|_{q+1}^{q+1}+\left\|\nabla u_{\tau}\right\|^{2}\right) d \tau \\
& =2 \int_{t_{1}}^{t_{2}} E(t) d t+D^{q+1}(t) .
\end{aligned}
$$

Then, by (28), we have

$$
\begin{aligned}
E(t) \leq & \left(\frac{1}{2} C+C_{3}\right) D^{2}(t)+D^{q+1}(t) \\
& +C_{4}\left[D(t)+D^{q}(t)\right] E^{\frac{1}{m+2}}(t) .
\end{aligned}
$$

Hence, we obtain

$$
E(t) \leq C_{5}\left[D^{2}(t)+D^{q+1}(t)+D^{\frac{m+2}{m+1}}(t)+D^{\frac{m+2}{m+1} q}(t)\right]
$$

Note that, since $E(t)$ is nonincreasing and $E(t) \geq 0$ on $[0, \infty)$,

$$
\begin{aligned}
D^{q+1}(t) & =E(t)-E(t+1) \\
& \leq E(0) .
\end{aligned}
$$

Thus, we have

$$
D(t) \leq E^{\frac{1}{q+1}}(0)
$$

It follows from (32) and (33) that

$$
\begin{aligned}
E(t) & \leq C_{5}\left[D^{\frac{m}{m+1}}(t)+D^{q-\frac{1}{m+1}}(t)+1+D^{\frac{(m+2)(q-1)}{m+1}}(t)\right] D^{\frac{m+2}{m+1}}(t) \\
& \leq C_{5}\left[E^{\frac{m}{(m+1)(q+1)}}(0)+E^{\left(q-\frac{1}{m+1}\right) \frac{1}{q+1}}(0)+1+E^{\frac{(m+2)(q-1)}{(m+1)(q+1)}}\right] D^{\frac{m+2}{m+1}}(t) \\
& =C_{6} D^{\frac{m+2}{m+1}}(t) .
\end{aligned}
$$

Thus, we get

$$
E^{1+\frac{(m+1) q-1}{m+2}}(t) \leq C_{7} D^{q+1}(t)
$$


Case 1: When $q=1$ and $m=0$ from (34), we obtain

$$
E(t) \leq C_{7} D^{2}(t)=C_{7}[E(t)-E(t+1)] .
$$

By Lemma 2, we get

$$
E(t) \leq E(0) e^{-w_{1}[t-1]^{+}},
$$

where $w_{1}=\ln \frac{C_{7}}{C_{7}-1}$.

Case 2: When $(m+1) q>1$, applying Lemma 2 to (34) yield

$$
E(t) \leq\left(E(0)^{-\alpha}+C_{7}^{-1} \alpha[t-1]^{+}\right)^{-\frac{1}{\alpha}}
$$

where $\alpha=\frac{(m+1) q-1}{m+2}$. The proof of Theorem 9 is completed.

\section{Blow up of solutions}

In this section, we deal with the blow up of the solution for the problem (1), when $q=1$. Let us begin by stating the following two lemmas, which will be used later.

Lemma 10 [14] Let us have $\delta>0$ and let $B(t) \in C^{2}(0, \infty)$ be a nonnegative function satisfying

$$
B^{\prime \prime}(t)-4(\delta+1) B^{\prime}(t)+4(\delta+1) B(t) \geq 0 .
$$

If

$$
B^{\prime}(0)>r_{2} B(0)+K_{0}
$$

with $r_{2}=2(\delta+1)-2 \sqrt{(\delta+1) \delta}$, then $B^{\prime}(t)>K_{0}$ for $t>0$, where $K_{0}$ is a constant.

Lemma 11 [14] If $H(t)$ is a nonincreasing function on $\left[t_{0}, \infty\right)$ and satisfies the differential inequality

$$
\left[H^{\prime}(t)\right]^{2} \geq a+b[H(t)]^{2+\frac{1}{\delta}} \quad \text { for } t \geq t_{0}
$$

where $a>0, b \in R$, then there exists a finite time $T^{*}$ such that

$$
\lim _{t \rightarrow T^{*-}} H(t)=0
$$

Upper bounds for $T^{*}$ are estimated as follows:

(i) If $b<0$ and $H\left(t_{0}\right)<\min \left\{1, \sqrt{-\frac{a}{b}}\right\}$ then

$$
T^{*} \leq t_{0}+\frac{1}{\sqrt{-b}} \ln \frac{\sqrt{-\frac{a}{b}}}{\sqrt{-\frac{a}{b}}-H\left(t_{0}\right)} .
$$


(ii) If $b=0$, then

$$
T^{*} \leq t_{0}+\frac{H\left(t_{0}\right)}{H^{\prime}\left(t_{0}\right)}
$$

(iii) If $b>0$, then

$$
T^{*} \leq \frac{H\left(t_{0}\right)}{\sqrt{a}} \quad \text { or } \quad T^{*} \leq t_{0}+2^{\frac{3 \delta+1}{2 \delta}} \frac{\delta c}{\sqrt{a}}\left[1-\left(1+c H\left(t_{0}\right)\right)^{-\frac{1}{2 \delta}}\right]
$$

where $c=\left(\frac{a}{b}\right)^{2+\frac{1}{\delta}}$.

Definition 12 A solution $u$ of (1) with $q=1$ is called blow up if there exists a finite time $T^{*}$ such that

$$
\lim _{t \rightarrow T^{*-}}\left[\int_{\Omega} u^{2} d x+\int_{0}^{t} \int_{\Omega}\left(u^{2}+|\nabla u|^{2}\right) d x d \tau\right]=\infty .
$$

Let

$$
a(t)=\int_{\Omega} u^{2} d x+\int_{0}^{t} \int_{\Omega}\left(u^{2}+|\nabla u|^{2}\right) d x d \tau \quad \text { for } t \geq 0 .
$$

Lemma 13 Assume $m+2<p+1<\frac{n(m+2)}{n-(m+2)}, m+2<n$, and that $m \leq 4 \delta \leq p-1$, then we have

$$
a^{\prime \prime}(t) \geq 4(\delta+1) \int_{\Omega} u_{t}^{2} d x-4(2 \delta+1) E(0)+4(2 \delta+1) \int_{0}^{t}\left(\left\|u_{t}\right\|^{2}+\left\|\nabla u_{t}\right\|^{2}\right) d \tau .
$$

Proof From (39), we have

$$
\begin{aligned}
a^{\prime}(t) & =2 \int_{\Omega} u u_{t} d x+\|u\|^{2}+\|\nabla u\|^{2} \\
a^{\prime \prime}(t) & =2 \int_{\Omega} u_{t}^{2} d x+2 \int_{\Omega} u u_{t t} d x+2 \int_{\Omega}\left(u u_{t}+\nabla u \nabla u_{t}\right) d x \\
& =2\left\|u_{t}\right\|^{2}-2\|\nabla u\|_{m+2}^{m+2}+2\|u\|_{p+1}^{p+1} .
\end{aligned}
$$

Then from (6) and (42), we have

$$
\begin{aligned}
a^{\prime \prime}(t)= & 4(\delta+1) \int_{\Omega} u_{t}^{2} d x-4(2 \delta+1) E(0)+4(2 \delta+1) \int_{0}^{t}\left(\left\|u_{t}\right\|^{2}+\left\|\nabla u_{t}\right\|^{2}\right) d \tau \\
& +\left(\frac{8 \delta+4}{m+2}-2\right)\|\nabla u\|_{m+2}^{m+2}+\left(2-\frac{8 \delta+4}{p+1}\right)\|u\|_{p+1}^{p+1} .
\end{aligned}
$$

Since $m \leq 4 \delta \leq p-1$, we obtain (40).

Lemma 14 Assume $m+2<p+1<\frac{n(m+2)}{n-(m+2)}, m+2<n$ and one of the following statements are satisfied:

(i) $E(0)<0$,

(ii) $E(0)=0$ and $\int_{\Omega} u_{0} u_{1} d x>0$, 
(iii) $E(0)>0$ and

$$
a^{\prime}(0)>r_{2}\left[a(0)+\frac{K_{1}}{4(\delta+1)}\right]+\left\|u_{0}\right\|^{2}
$$

holds.

Then $a^{\prime}(t)>\left\|u_{0}\right\|^{2}$ for $t>t^{*}$, where $t_{0}=t^{*}$ is given by (44) in case (i) and $t_{0}=0$ in cases (ii) and (iii).

Here $K_{1}$ and $t^{*}$ are defined in (48) and (44), respectively.

Proof (i) If $E(0)<0$, then from (40), we have

$$
a^{\prime}(t) \geq a^{\prime}(0)-4(2 \delta+1) E(0) t, \quad t \geq 0 .
$$

Thus we get $a^{\prime}(t)>\left\|u_{0}\right\|^{2}$ for $t>t^{*}$, where

$$
t^{*}=\max \left\{\frac{a^{\prime}(t)-\left\|u_{0}\right\|^{2}}{4(2 \delta+1) E(0)}, 0\right\} .
$$

(ii) If $E(0)=0$ and $\int_{\Omega} u_{0} u_{1} d x>0$, then $a^{\prime \prime}(t) \geq 0$ for $t \geq 0$. We have $a^{\prime}(t)>\left\|u_{0}\right\|^{2}, t \geq 0$.

(iii) If $E(0)>0$, we first note that

$$
2 \int_{0}^{t} \int_{\Omega} u u_{t} d x d \tau=\|u\|^{2}-\left\|u_{0}\right\|^{2}
$$

By the Hölder inequality and the Young inequality, we have

$$
\|u\|^{2} \leq\left\|u_{0}\right\|^{2}+\int_{0}^{t}\|u\|^{2} d \tau+\int_{0}^{t}\left\|u_{t}\right\|^{2} d \tau
$$

By the Hölder inequality, the Young inequality, and (46), we have

$$
a^{\prime}(t) \leq a(t)+\left\|u_{0}\right\|^{2}+\int_{\Omega} u_{t}^{2} d x+\int_{0}^{t}\left\|u_{t}\right\|^{2} d \tau
$$

Hence, by (40) and (47), we obtain

$$
a^{\prime \prime}(t)-4(\delta+1) a^{\prime}(t)+\left\|u_{0}\right\|^{2} a(t)+K_{1} \geq 0,
$$

where

$$
K_{1}=4(2 \delta+1) E(0)+4(\delta+1)\left\|u_{0}\right\|^{2} .
$$

Let

$$
b(t)=a(t)+\frac{K_{1}}{4(\delta+1)}, \quad t>0 .
$$

Then $b(t)$ satisfies Lemma 10. Consequently, we get from (43) $a^{\prime}(t)>\left\|u_{0}\right\|^{2}, t>0$, where $r_{2}$ is given in Lemma 10 . 
Theorem 15 Assume $m+2<p+1<\frac{n(m+2)}{n-(m+2)}, m+2<n$ and one of the following statements are satisfied:

(i) $E(0)<0$,

(ii) $E(0)=0$ and $\int_{\Omega} u_{0} u_{1} d x>0$,

(iii) $0<E(0)<\frac{\left(a^{\prime}\left(t_{0}\right)-\left\|u_{0}\right\|^{2}\right)^{2}}{8\left[a\left(t_{0}\right)+\left(T_{1}-t_{0}\right)\left\|u_{0}\right\|^{2}\right]}$ and (43) holds.

Then the solution u blow up in finite time $T^{*}$ in the case of (38). In case (i),

$$
T^{*} \leq t_{0}-\frac{H\left(t_{0}\right)}{H^{\prime}\left(t_{0}\right)}
$$

Furthermore, if $H\left(t_{0}\right)<\min \left\{1, \sqrt{-\frac{a}{b}}\right\}$, we have

$$
T^{*} \leq t_{0}+\frac{1}{\sqrt{-b}} \ln \frac{\sqrt{-\frac{a}{b}}}{\sqrt{-\frac{a}{b}}-H\left(t_{0}\right)},
$$

where

$$
\begin{aligned}
& a=\delta^{2} H^{2+\frac{2}{\delta}}\left(t_{0}\right)\left[\left(a^{\prime}\left(t_{0}\right)-\left\|u_{0}\right\|^{2}\right)^{2}-8 E(0) H^{-\frac{1}{\delta}}\left(t_{0}\right)\right]>0, \\
& b=8 \delta^{2} E(0) .
\end{aligned}
$$

In case (ii),

$$
T^{*} \leq t_{0}-\frac{H\left(t_{0}\right)}{H^{\prime}\left(t_{0}\right)}
$$

In case (iii),

$$
T^{*} \leq \frac{H\left(t_{0}\right)}{\sqrt{a}} \quad \text { or } \quad T^{*} \leq t_{0}+2^{\frac{3 \delta+1}{2 \delta}}\left(\frac{a}{b}\right)^{2+\frac{1}{\delta}} \frac{\delta}{\sqrt{a}}\left\{1-\left[1+\left(\frac{a}{b}\right)^{2+\frac{1}{\delta}} H\left(t_{0}\right)\right]^{-\frac{1}{2 \delta}}\right\}
$$

where $a$ and $b$ are given; see (51), (52).

Proof Let

$$
H(t)=\left[a(t)+\left(T_{1}-t\right)\left\|u_{0}\right\|^{2}\right]^{-\delta} \quad \text { for } t \in\left[0, T_{1}\right]
$$

where $T_{1}>0$ is a certain constant which will be specified later. Then we get

$$
\begin{aligned}
H^{\prime}(t)= & -\delta\left[a(t)+\left(T_{1}-t\right)\left\|u_{0}\right\|^{2}\right]^{-\delta-1}\left[a^{\prime}(t)-\left\|u_{0}\right\|^{2}\right] \\
= & -\delta H^{1+\frac{1}{\delta}}(t)\left[a^{\prime}(t)-\left\|u_{0}\right\|^{2}\right], \\
H^{\prime \prime}(t)= & -\delta H^{1+\frac{2}{\delta}}(t) a^{\prime \prime}(t)\left[a(t)+\left(T_{1}-t\right)\left\|u_{0}\right\|^{2}\right] \\
& +\delta H^{1+\frac{2}{\delta}}(t)(1+\delta)\left[a^{\prime}(t)-\left\|u_{0}\right\|^{2}\right]^{2}
\end{aligned}
$$

and

$$
H^{\prime \prime}(t)=-\delta H^{1+\frac{2}{\delta}}(t) V(t),
$$


where

$$
V(t)=a^{\prime \prime}(t)\left[a(t)+\left(T_{1}-t\right)\left\|u_{0}\right\|^{2}\right]-(1+\delta)\left[a^{\prime}(t)-\left\|u_{0}\right\|^{2}\right]^{2}
$$

For simplicity of the calculation, we define

$$
\begin{aligned}
& P_{u}=\int_{\Omega} u^{2} d x, \quad R_{u}=\int_{\Omega} u_{t}^{2} d x, \\
& Q_{u}=\int_{0}^{t}\|u\|^{2} d t, \quad S_{u}=\int_{0}^{t}\left\|u_{t}\right\|^{2} d t .
\end{aligned}
$$

From (41), (45), and the Hölder inequality, we get

$$
\begin{aligned}
a^{\prime}(t) & =2 \int_{\Omega} u u_{t} d x+\left\|u_{0}\right\|^{2}+2 \int_{0}^{t} \int_{\Omega} u u_{t} d x d t \\
& \leq 2\left(\sqrt{R_{u} P_{u}}+\sqrt{Q_{u} S_{u}}\right)+\left\|u_{0}\right\|^{2} .
\end{aligned}
$$

If case (i) or (ii) holds, by (40) we have

$$
a^{\prime \prime}(t) \geq(-4-8 \delta) E(0)+4(1+\delta)\left(R_{u}+S_{u}\right) .
$$

Thus, from (59)-(61) and (55), we obtain

$$
\begin{aligned}
V(t) \geq[ & \left.(-4-8 \delta) E(0)+4(1+\delta)\left(R_{u}+S_{u}\right)\right] H^{-\frac{1}{\delta}}(t) \\
& -4(1+\delta)\left(\sqrt{R_{u} P_{u}}+\sqrt{Q_{u} S_{u}}\right)^{2} .
\end{aligned}
$$

From (39),

$$
a(t)=\int_{\Omega} u^{2} d x+\int_{0}^{t} \int_{\Omega} u^{2} d x d s=P_{u}
$$

and (55), we get

$$
V(t) \geq(-4-8 \delta) E(0) H^{-\frac{1}{\delta}}(t)+4(1+\delta)\left[\left(R_{u}+S_{u}\right)\left(T_{1}-t\right)\left\|u_{0}\right\|^{2}+\Theta(t)\right],
$$

where

$$
\Theta(t)=\left(R_{u}+S_{u}\right)\left(P_{u}+Q_{u}\right)-\left(\sqrt{R_{u} P_{u}}+\sqrt{Q_{u} S_{u}}\right)^{2} .
$$

By the Schwarz inequality, and $\Theta(t)$ being nonnegative, we have

$$
V(t) \geq(-4-8 \delta) E(0) H^{-\frac{1}{\delta}}(t), \quad t \geq t_{0} .
$$

Therefore, by (58) and (62), we get

$$
H^{\prime \prime}(t) \leq 4 \delta(1+2 \delta) E(0) H^{1+\frac{1}{\delta}}(t), \quad t \geq t_{0} .
$$


By Lemma 13, we know that $H^{\prime}(t)<0$ for $t \geq t_{0}$. Multiplying (63) by $H^{\prime}(t)$ and integrating it from $t_{0}$ to $t$, we get

$$
H^{\prime 2}(t) \geq a+b H^{2+\frac{1}{\delta}}(t)
$$

for $t \geq t_{0}$, where $a, b$ are defined in (51) and (52) respectively.

If case (iii) holds, by the steps of case (i), we get $a>0$ if and only if

$$
E(0)<\frac{\left(a^{\prime}\left(t_{0}\right)-\left\|u_{0}\right\|^{2}\right)^{2}}{8\left[a\left(t_{0}\right)+\left(T_{1}-t_{0}\right)\left\|u_{0}\right\|^{2}\right]} .
$$

Then by Lemma 11, there exists a finite time $T^{*}$ such that $\lim _{t \rightarrow T^{*-}} H(t)=0$ and the upper bound of $T^{*}$ is estimated according to the sign of $E(0)$. This means that (38) holds.

\section{Competing interests}

The author declares that they have no competing interests.

\section{Acknowledgements}

The author would like to thank the anonymous referees for their valuable comments and suggestions.

Received: 13 December 2014 Accepted: 18 July 2015 Published online: 01 August 2015

\section{References}

1. Andrews, G: On the existence of solutions to the equation $u_{t t}-u_{x x t}=\sigma\left(u_{x}\right)_{x}$. J. Differ. Equ. 35, 200-231 (1980)

2. Yang, Z: Existence and asymptotic behavior of solutions for a class of quasilinear evolution equations with nonlinear damping and source terms. Math. Methods Appl. Sci. 25, 795-814 (2002)

3. Gerbi, S, Houari, BS: Exponential decay for solutions to semilinear damped wave equation. Discrete Contin. Dyn. Syst., Ser. B 5(3), 559-566 (2012)

4. Chen, H, Liu, G: Global existence, uniform decay and exponential growth for a class of semilinear wave equation with strong damping. Acta Math. Sci., Ser. B 33(1), 41-58 (2013)

5. Gazzola, F, Squassina, M: Global solutions and finite time blow up for damped semilinear wave equations. Ann. Inst. Henri Poincaré, Anal. Non Linéaire 23, 185-207 (2006)

6. Georgiev, V, Todorova, G: Existence of a solution of the wave equation with nonlinear damping and source terms. J. Differ. Equ. 109(2), 295-308 (1994)

7. Levine, HA: Instability and nonexistence of global solutions to nonlinear wave equations of the form $P u_{t t}=A u+F(u)$. Trans. Am. Math. Soc. 192, 1-21 (1974)

8. Levine, HA: Some additional remarks on the nonexistence of global solutions to nonlinear wave equations. SIAM J. Math. Anal. 5, 138-146 (1974)

9. Messaoudi, SA: Blow up in a nonlinearly damped wave equation. Math. Nachr. 231, 105-111 (2001)

10. $\mathrm{Xu}, \mathrm{R}$, Shen, J: Some generalized results for global well-posedness for wave equations with damping and source terms. Math. Comput. Simul. 80, 804-807 (2009)

11. Vitillaro, E: Global nonexistence theorems for a class of evolution equations with dissipation. Arch. Ration. Mech. Anal. 149, 155-182 (1999)

12. Messaoudi, SA: On the decay of solutions for a class of quasilinear hyperbolic equations with nonlinear damping and source terms. Math. Methods Appl. Sci. 28, 1819-1828 (2005)

13. $\mathrm{Wu}, \mathrm{Y}, \mathrm{Xue}, \mathrm{X}$ : Uniform decay rate estimates for a class of quasilinear hyperbolic equations with nonlinear damping and source terms. Appl. Anal. 92(6), 1169-1178 (2013)

14. Li, MR, Tsai, LY: Existence and nonexistence of global solutions of some system of semilinear wave equations. Nonlinear Anal. 54(8), 1397-1415 (2003)

15. Adams, RA, Fournier, JJF: Sobolev Spaces. Academic Press, San Diego (2003)

16. Nakao, M: Asymptotic stability of the bounded or almost periodic solution of the wave equation with nonlinear dissipative term. J. Math. Anal. Appl. 58(2), 336-343 (1977)

17. Pişkin, E, Polat, N: Global existence, decay and blow up solutions for coupled nonlinear wave equations with damping and source terms. Turk. J. Math. 37, 633-651 (2013)

18. Ye, Y: Existence and decay estimate of global solutions to systems of nonlinear wave equations with damping and source terms. Abstr. Appl. Anal. 2013, Article ID 903625 (2013) 